\title{
Binding of smoothelin-like 1 to tropomyosin and calmodulin is mutually exclusive and regulated by phosphorylation
}

\author{
Annegret Ulke-Lemée ${ }^{1}$, David Hao Sun ${ }^{1}$, Hiroaki Ishida ${ }^{2}$, Hans J. Vogel ${ }^{2}$ and Justin A. MacDonald ${ }^{\text {** }}$
}

\begin{abstract}
Background: The smoothelin-like 1 protein (SMTNL1) can associate with tropomyosin (Tpm) and calmodulin (CaM), two proteins essential to the smooth muscle contractile process. SMTNL1 is phosphorylated at Ser301 by protein kinase A during calcium desensitization in smooth muscle, yet the effect of SMTNL1 phosphorylation on Tpm- and CaM-binding has yet to be investigated.

Results: Using pull down studies with Tpm-Sepharose and CaM-Sepharose, we examined the interplay between Tpm binding, CaM binding, phosphorylation of SMTNL1 and calcium concentration. Phosphorylation greatly enhanced the ability of SMTNL1 to associate with Tpm in vitro; surface plasmon resonance yielded a 10-fold enhancement in $K_{\mathrm{D}}$ value with phosphorylation. The effect on CaM binding is more complex and varies with the availability of calcium.
\end{abstract}

Conclusions: Combining both CaM and Tpm with SMTNL1 shows that the binding to both is mutually exclusive.

Keywords: SMTNL1, CHASM, Smooth muscle, Thin filament, Protein binding

\section{Background}

Smoothelin-like 1 protein (SMTNL1), also termed calponin homology-associated with smooth muscle (CHASM) protein, was originally identified as a protein phosphorylated in response to cyclic nucleotide-dependent protein kinase (i.e., protein kinase A (PKA) and protein kinase G (PKG)) activation during calcium desensitization of gastrointestinal smooth muscle [1]. Additional studies have revealed a role for SMTNL1 in vascular smooth muscle contractile activity, as well as cardiovascular and skeletal muscle adaptation to exercise, development and pregnancy [2-6].

The ubiquitous calmodulin $(\mathrm{CaM})$ is the primary cellular signal transducer that provides spatial and temporal responses to changes in intracellular calcium levels [7]. CaM provides signaling by association with specific binding sites in target proteins, both in its calcium-saturated (Ca-CaM) or its calcium-free (apo-CaM) form [8, 9]. We have previously reported that SMTNL1 possesses two CaM-binding sites: a classic CaM-binding domain (CBD1, amino acids

\footnotetext{
* Correspondence: jmacdo@ucalgary.ca

${ }^{1}$ Department of Biochemistry \& Molecular Biology, University of Calgary, Cumming School of Medicine, 3280 Hospital Drive NW, Calgary, AB T2N 4Z6, Canada Full list of author information is available at the end of the article
}

310-325) in the center of the protein as well as an IQmotif that serves as an apo-CaM-binding site (CDB2, amino acids 439-457) located in the carboxy-terminal calponin homology domain [10-12]. SMTNL1 was established as a bona fide CaM-binding protein within aortic A7r5 smooth muscle cells using the proximity ligation assay. In this regard, CBD1 is thought to provide the majority of CaM-binding in situ; however, CBD2 may contribute cooperatively to binding [12].

A second binding partner of SMTNL1 has also been identified in smooth muscle. Tropomyosin (Tpm), a muscle protein that aids in the stabilization of actin filaments, is incorporated as super-helical polymers along smooth muscle thin filaments and contribute to the regulation of actin-myosin cross bridge cycling during smooth muscle contraction [13-15]. In smooth muscle, two Tpm isoforms (Tpm1.4 and Tpm2.1; previously identified as Tmsm- $\alpha / \operatorname{Tm} 6$ and Tmsm- $\beta / \operatorname{Tm} 1 \beta$, respectively [16]) are predominantly expressed [17]. SMTNL1 was shown to associate with smooth muscle Tpm $\alpha / \beta$ dimers with a binding surface comprising of its $\mathrm{N}$-terminal intrinsically-disordered region and the C-terminal calponin homology domain [18]. 
Previous in vitro studies have defined SMTNL1 as a Tpm- and CaM-binding protein [10-12, 18, 19]. Moreover, the phosphorylation of SMTNL1 was identified during calcium desensitization of smooth muscle $[1,5]$. However, the effect of phosphorylation on the ability of SMTNL1 to associate with Tpm and/or CaM has not been investigated. Intriguingly, the binding domains for $\mathrm{CaM}$ (CBD1 and CBD2) are localized in close vicinity to the Tpm-binding surface on the SMTNL1 protein. In addition, the Ser301 phosphorylation site targeted by cyclic nucleotide-dependent protein kinases (i.e., PKA and PKG) is located near CBD1 and within the Tpmbinding region $[11,18]$. This led us to inquire about the interplay of SMTNL1 phosphorylation and calcium levels on the multi-functionality of Tpm- and CaMbinding. Herein, we demonstrate that phosphorylation of Ser301 greatly enhanced the ability of SMTNL1 to associate with smooth muscle Tpm in vitro. The effect of Ser301 phosphorylation on CaM binding was more complex and varied with the availability of calcium. Combining both $\mathrm{CaM}$ and $\mathrm{Tpm}$ revealed that binding with SMTNL1 was mutually exclusive.

\section{Methods}

\section{Materials}

PreScission Protease, glutathione-Sepharose and CNBractivated Sepharose were from GE Healthcare (Piscataway, NJ). The Phos-tag acrylamide reagent was from NARD Chemicals (Kobe City, Japan). Smooth muscle Tpm was a purified $\alpha / \beta$ heterodimer of chicken gizzard Tpm1.4 $4_{\text {sm(a.a.b.d) }}$ (equivalent to Tm6 or Tmsm- $\alpha$ ) and Tpm2.1 $1_{\text {sm(a.b.a.d) }}$ (equivalent to TM-1, $\beta$ Tm or Tmsm- $\beta$ ) [16] and was provided by Dr. Michael Walsh (University of Calgary). CaM was provided by Dr. Hans Vogel (University of Calgary). PKA was purified from bovine heart using the method described in [20].

\section{Expression and Purification of SMTNL1}

A clone comprising the Tpm-binding region of SMTNL1 (SMTNL1-TMB, amino acids 195-459; NP_077192) was described previously [18, 19]. SMTNL1-TMB was expressed as a glutathione $S$-transferase (GST)-fusion protein in $E$. coli and purified with glutathioneSepharose. The fusion protein was cleaved with PreScission Protease, and GST was removed by an additional pass over glutathione-Sepharose along with a final cleanup using MonoQ anion exchange chromatography. In some cases, SMTNL1-TMB was phosphorylated with PKA at a molar ratio of 500:1 (SMTNL1: PKA) in a buffer consisting of $2 \mathrm{mM} \mathrm{MgCl} 2,0.2 \mathrm{mM}$ ATP, $25 \mathrm{mM}$ HEPES and $150 \mathrm{mM} \mathrm{NaCl}$. The phosphorylated protein was buffer-exchanged and concentrated using a $3 \mathrm{kDa}$ cutoff centrifugation filter unit (EMD Millipore, Billerica, MA).

\section{Tpm-Sepharose binding assay}

Purified smooth muscle Tpm or Tris- $\mathrm{HCl}$ (as control) was covalently bound to $\mathrm{CNBr}$-activated Sepharose following the manufacturers' instructions and as described in [19]. SMTNL1-TMB $(200 \mu \mathrm{g})$ was incubated with $40 \mu \mathrm{L}$ of TpmSepharose or Control-Sepharose in binding buffer $(20 \mathrm{mM}$ Tris- $\mathrm{HCl}, \mathrm{pH} 7.5$ with $0.1 \%$ (v/v) $\beta$-mercaptoethanol) for $2 \mathrm{~h}$ at $4{ }^{\circ} \mathrm{C}$ and then washed extensively with the same buffer supplemented with $150 \mathrm{mM} \mathrm{NaCl}$. Bound protein was eluted with SDS-PAGE loading buffer, separated by gel electrophoresis, and detected by Coomassie stain.

\section{Calmodulin-Sepharose binding assay}

Calmodulin (CaM)-Sepharose pull-down experiments were completed as described previously [11]. Briefly, SMTNL1TMB $(200 \mu \mathrm{g})$ was incubated with $40 \mu \mathrm{L}$ of CaM-Sepharose in binding buffer (20 mM HEPES, pH 7.0 in the presence of $5 \mathrm{mM} \mathrm{CaCl}_{2}$ (Ca-CaM) or $1 \mathrm{mM}$ EDTA (apo-CaM)). After incubation for $1 \mathrm{~h}$ at $4{ }^{\circ} \mathrm{C}$, the CaM-Sepharose was washed extensively with the same buffer supplemented with $150 \mathrm{mM} \mathrm{NaCl}$. Bound SMTNL1-TMB protein was eluted with SDS-PAGE loading buffer, separated by gel electrophoresis, and detected by Coomassie stain. In some experiments, SMTNL1-TMB was premixed with Tpm in different molar ratios, allowed to form complexes for $1 \mathrm{~h}$ at $4{ }^{\circ} \mathrm{C}$ and then added to the CaM-Sepharose.

\section{Surface Plasmon Resonance (SPR)}

The binding between Tpm and SMTNL1-TMB with or without phosphorylation was evaluated by SPR using a BIAcore X100 instrument (GE Healthcare). Purified Tpm was immobilized via amine-coupling onto a CM5 sensor chip (GE Healthcare). The running buffer contained $20 \mathrm{mM}$ HEPES $\mathrm{pH}$ 7.5, $100 \mathrm{mM} \mathrm{KCl,} 1 \mathrm{mM}$ DTT, and $0.005 \%(\mathrm{v} / \mathrm{v})$ Tween-20. Five concentrations of SMTNL1-TMB from 0.12 to $10 \mu \mathrm{M}$ were prepared by serial dilution and were injected at a flow rate of $30 \mu \mathrm{L} /$ min with a contact time of $1 \mathrm{~min}$ at $25{ }^{\circ} \mathrm{C}$. The chip was regenerated by injecting $1 \mathrm{M} \mathrm{NaCl}$ for 4 min followed by glycine- $\mathrm{HCl}(10 \mathrm{mM}, \mathrm{pH} 3.0)$ for $1 \mathrm{~min}$. Each experiment was repeated three times $(n=3)$ to obtain a standard error (SE). The BIAevaluation software 2.0 (GE Healthcare) was used to analyze the SPR sensorgrams and to obtain the dissociation constants $(\mathrm{Kd})$.

\section{Data analysis}

Values are presented as the mean \pm S.E.M., with $n$ indicating the number of independent experiments. Data were analyzed with two-tailed Student's $t$ test, or for comparison of multiple groups, with one-way analysis of variance (ANOVA) and Tukey's post hoc test. Differences were considered to be statistically significant for $p<0.05$. All statistical analyses were performed using the GraphPad Prism 6.0 program. 


\section{Results}

\section{Phosphorylation of SMTNL1 by PKA enhances} Tpm-binding potential

Full-length SMTNL1 protein exhibits reduced stability that complicates conclusions from in vitro binding experiments. A truncated form of SMTNL1 (i.e., SMTNL1-TMB) was used since this clone is stable under the conditions and times used; furthermore, SMTNL1-TMB contains all known functional elements of SMTNL1 (e.g., CBD1, CBD2, calponin-homology $(\mathrm{CH})$ domain, Tpm-binding regions and Ser301 phosphorylation site; Fig. 1a). SMTNL1-TMB could be effectively phosphorylated with PKA; the phosphorylation stoichiometry was assessed with Phos-tag SDS-PAGE and judged to be complete (i.e., $1 \mathrm{~mol}$ phosphate $/ \mathrm{mol}$ SMTNL1) after $16 \mathrm{~h}$ incubation at $4{ }^{\circ} \mathrm{C}$ (Fig. 1b). The binding of SMTNL1-TMB to Tpm was examined in pull-down assays with purified smooth muscle Tpm (mixture of Tpm1.4 and Tpm2.1 isoforms) immobilized to Sepharose resin (Tpm-Sepharose). SMTNL1-TMB was recovered from Tpm-Sepharose while no interaction with ControlSepharose was observed (Fig. 1c). Some Tpm could be released from the Sepharose column with application of SDS elution buffer and boiling. This was anticipated given that smooth muscle Tpm exists as coiled-coil heterodimers [21], and it was unlikely that all Tpm molecules could be covalently coupled to the $\mathrm{CNBr}$-Sepharose resin. Approximately 2-fold more phosphorylated SMTNL1-TMB could be recovered from pull-down assays with Tpm-Sepharose (Fig. 1d) when compared with unphosphorylated SMTNL1-TMB. Binding constants for a kinetic series, $0-10 \mu \mathrm{M}$ phosphorylated SMTNL1-TMB provided a $K_{\mathrm{D}}$ of $3.2 \times 10^{-7} \mathrm{M}$ with biophysical analyses by SPR (Additional file 1: Figure S1), a response that was enhanced 10-fold when compared to unphosphorylated SMTNL1-TMB $\left(3.0 \times 10^{-6} \mathrm{M}\right)$. The SPR assays provided a similar $K_{\mathrm{D}}$ for unphosphorylated SMTNL1 and Tpm as previously defined by isothermal titration calorimetry (ITC) assays [19]. We previously reported that the SMTNL1 binding surface for Tpm included a central portion of the intrinsically-disordered region, located proximal to the Ser301 phosphorylation site, as well as a surface of the calponin homology $(\mathrm{CH})$ domain located at the $C$-terminus [18]. So, it is not unexpected that phosphorylation at Ser301 could alter the electrostatic properties and/or the intramolecular conformation of the Tpm-binding surface on SMTNL1.

\section{Phosphorylation of SMTNL1-TMB influences binding to CaM-Sepharose}

The Ser301 phosphorylation site of SMTNL1 is located in close proximity to the $\mathrm{Ca}-\mathrm{CaM}$ binding domain (CBD1, amino acids 310-325 [11]). It was expected that the phosphorylation of SMTNL1-TMB by PKA would impact the association with $\mathrm{Ca}-\mathrm{CaM}$ and not with apo-
CaM, since the binding of the latter was linked to an IQ-domain at the C-terminus (CBD2, amino acids 439457 [10]). Indeed, we observed reductions in Ca-CaMSepharose binding for phosphorylated SMTNL1-TMB, with recovery of approximately $30 \%$ less bound material (Fig. 2a). Surprisingly, the phosphorylation of SMTNL1TMB protein also impacted upon binding to apo-CaMSepharose (Fig. 2b). Although a general reduction in SMTNL1-TMB bound to CaM was observed in the apo condition (i.e., $\sim 60 \%$ less SMTNL1-TMB recovered on the resin), the recovery of phosphorylated SMTNL1TMB from the resin was further reduced to approximately $20 \%$ of the unphosphorylated material (Fig. 2b). We have previously demonstrated that the binding of SMTNL1-TMB to CaM was influenced by calcium, with SMTNL1 binding to CaM-Sepharose decreased by approximately $50 \%$ in the apo-calcium condition [11].

\section{Phosphorylation and calcium influence SMTNL1-TMB binding to CaM-Sepharose in the presence of Tpm} Equimolar amounts of SMTNL1-TMB and Tpm proteins were pre-incubated and then applied to CaMSepharose so that competition between CaM and Tpm for binding to SMTNL1-TMB could be assessed. Binding of SMTNL1-TMB to apo-CaM-Sepharose was reduced by approximately $60 \%$ by the addition of $\mathrm{Tpm}$ to SMTNL1-TMB prior to capture with the resin (Fig. 2b). These data indicate that Tpm could interfere with the apo-CaM binding properties of SMTNL1-TMB, likely due to the proximity of the two binding sites within the calponin homology $(\mathrm{CH})$ domain. Furthermore, we can conclude that SMTNL1-TMB interacts with Tpm more strongly than with apo-CaM. This was not unexpected as the interaction of SMTNL1 with apo-CaM is weak (Kd $\left.\sim 10^{-6} \mathrm{M}[10]\right)$. The results of the binding assays were not affected by the order of addition of proteins since pre-incubating SMTNL1-TMB with CaMSepharose followed by competition with Tpm showed the same reduction in binding (data not shown). The subsequent addition of Tpm to the mixture of phosphorylated SMTNL1 and apo-CaM-Sepharose, shown in Fig. 2b, further decreased binding potential to barely detectably levels. When investigating interactions with $\mathrm{Ca}-\mathrm{CaM}$, the binding of unphosphorylated SMTNL1TMB to Ca-CaM-Sepharose was not significantly influenced by the addition of equimolar amounts of Tpm (Fig. 2a). However, the addition of Tpm did significantly reduce the recovery of phosphorylated SMTNL-TMB on the $\mathrm{Ca}-\mathrm{CaM}-\mathrm{Sepharose}$ resin.

\section{SMTNL1 phosphorylation diminishes Ca-CaM-binding potential under conditions of high Tpm content} The intracellular Tpm concentration is predicted to be significantly higher than that of SMTNL1, as judged 


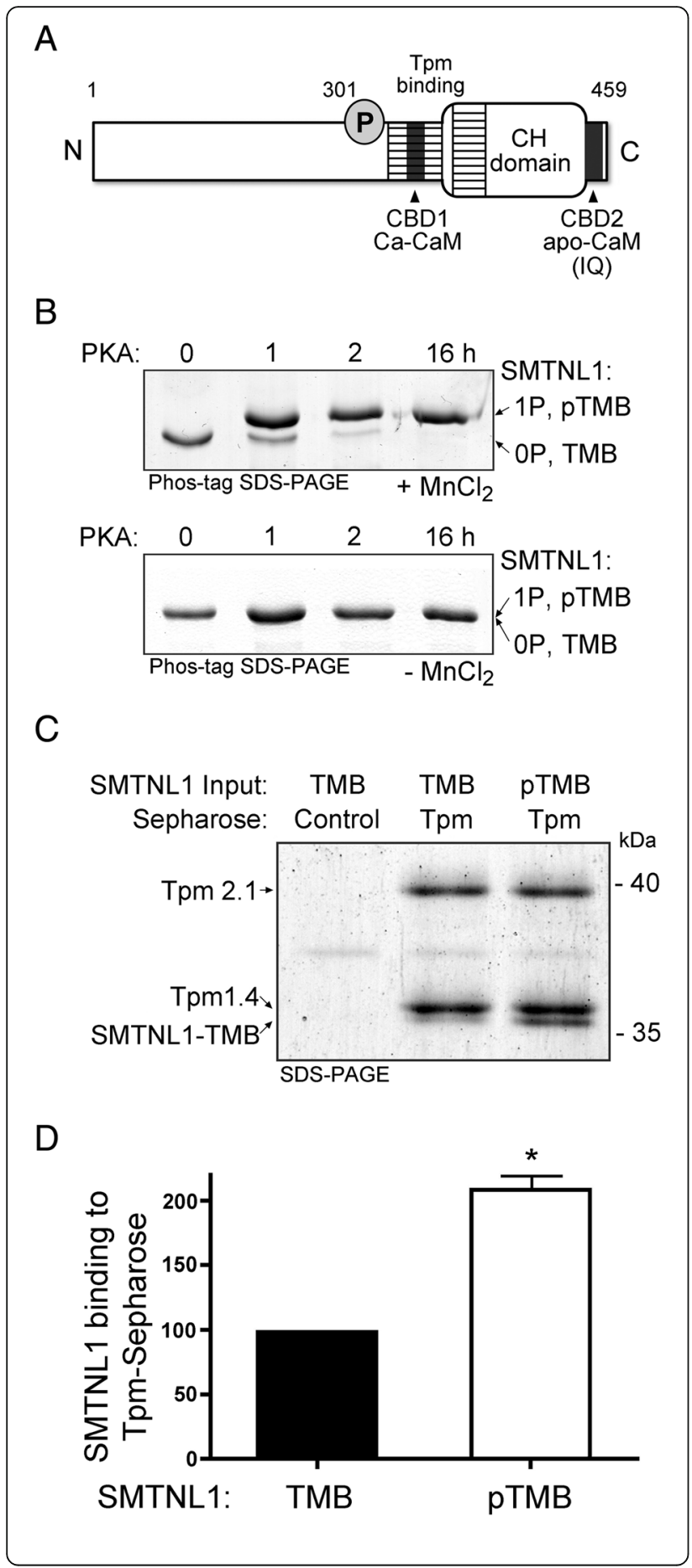

Fig. 1 The phosphorylation of SMTNL1 by PKA alters its tropomyosin-binding potential. a The SMTNL1 protein contains: a C-terminal calponin homology $(\mathrm{CH})$ domain, Ser301 PKAphosphorylation site, Ca-calmodulin (CaM)-binding domain (CBD1), apo-CaM-binding domain (CBD2), and tropomyosin (Tpm)-binding domain (indicated as the hatched area). $\mathbf{b}$ Purified recombinant SMTNL1-TMB was incubated with PKA as described in the Methods section. Samples were withdrawn at the indicated times and subjected to Phos-tag SDS-PAGE; two discrete bands representing unphosphorylated SMTNL1-TMB (OP, TMB) or SMTNL1-TMB phosphorylated at Ser301 (1P, pTMB) were detected by Coomassie stain. Samples subjected to Phos-tag SDS-PAGE in the absence of $\mathrm{MnCl}_{2}$ confirmed the shift in band migration to be a result of phosphorylation. c Unphosphorylated or phosphorylated SMTNL1-TMB $(200 \mu \mathrm{g})$ was incubated with $40 \mu \mathrm{L}$ of Tpm-Sepharose $(\alpha / \beta$-heterodimer: Tpm1.4/Tpm2.1). Bound SMTNL1-TMB was eluted with boiling $0.1 \%$ SDS solution and detected with Coomassie staining of SDS-PAGE gels. $\mathbf{d}$ The SMTNL1-TMB bands were quantified by densitometry, and binding to Tpm-Sepharose was expressed as percentage of the SMTNL1-TMB binding found for the unphosphorylated state. All experiments are $n=3-5$ and were analyzed by Student's $t$-test. *- Significantly different from unphosphorylated SMTNL1-TMB, $p<0.05$

from the relative staining intensities of 2D-SDS-PAGE gels [1]. So, the incubation of SMTNL1-TMB with different molar ratios of $\mathrm{Tpm}$ prior to the addition of CaM-Sepharose was used to reveal additional information about the character of the protein complex formation. Increasing the amounts of Tpm resulted in a notable decrease in the binding of SMTNL1-TMB to apo-CaM-Sepharose (Fig. 3a). Moreover, there was minimal influence of increased Tpm on the binding of phosphorylated SMTNL1-TMB in the apo-CaM condition (Fig. 3b). The small inhibitory effect of increasing Tpm concentration on the amount of SMTNL1-TMB retained on $\mathrm{Ca}-\mathrm{CaM}$ Sepharose suggests a greater affinity of SMTNL1-TMB for Ca-CaM over Tpm (Fig. 3c). However, distinct effects were observed for $\mathrm{Ca}-\mathrm{CaM}$ binding of phosphorylated SMTNL1-TMB under conditions of high Tpm (Fig. 3d). In this case, the phosphorylation of SMTNL1-TMB suppressed Ca-CaM binding potential in favour of Tpm.

\section{Discussion}

Phosphorylation events are known to regulate binding of $\mathrm{CaM}$ to target proteins. Several examples of phosphorylation within or near CaM-binding domains (CBDs) have been reported in the literature, including but not limited to smooth muscle myosin light chain kinase [22], MARKS protein [23], plasma membrane $\mathrm{Ca}^{2+}$-ATPase [24], endothelial nitric oxide synthase (eNOS) [25], and the $\mathrm{Ca}^{2+}$-dependent $\mathrm{K}^{+}$-channel [26]. CaM binds to its targets by wrapping around a short amphipathic helix within the CBD $[8,9]$, thus the phosphorylation of residues within a CBD can disrupt CaM interactions even if the Ser/Thr residues subject to phosphorylation are 

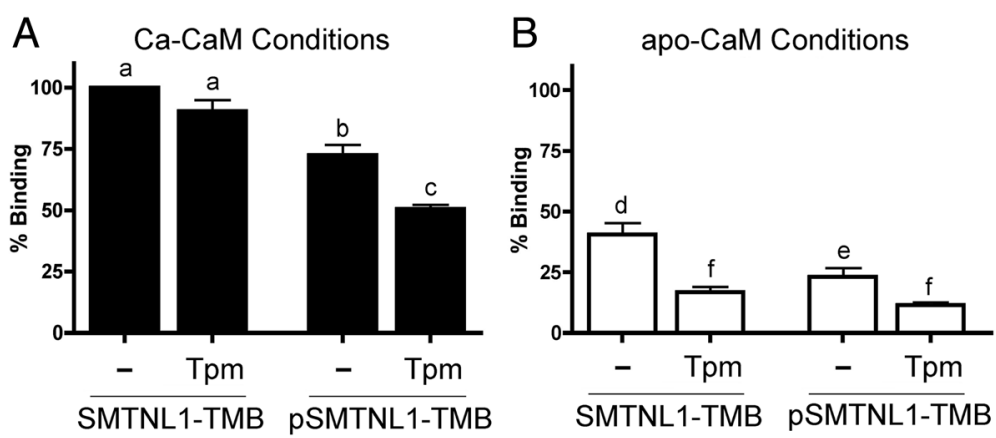

Fig. 2 Interdependency of SMTNL1 phosphorylation, calcium and tropomyosin on calmodulin-binding. SMTNL1-TMB (200 $\mu \mathrm{g}, 7 \mathrm{nmol})$ was incubated with an equimolar amount of Tpm. The mixture was then incubated with CaM-Sepharose (40 $\mu \mathrm{L}$; ligand density of $\sim 10-14 \mu \mathrm{mol} /$ $\mathrm{mL}$ ) in the presence $(\mathbf{a} ; \mathrm{Ca}-\mathrm{CaM}, 5 \mathrm{mM} \mathrm{CaCl}$ ) or absence ( $\mathbf{b}$; apo-CaM, $1 \mathrm{mM}$ EDTA) of calcium. Some experiments were completed with SMTNL1-TMB that had been previously phosphorylated with PKA. After washing, the retention of SMTNL1-TMB or phosphorylated SMTNL1TMB was analyzed. The band densities were quantified and binding to CaM-Sepharose expressed as percentage of SMTNL1-TMB recovered under maximal binding conditions (i.e., Ca-CaM in the absence of Tpm). All experiments are $n=3-5$ and were analyzed by one-way ANOVA with Tukey's post hoc analysis. Different letters indicate significant differences among groups (a,b,c: comparison among all Ca-CaM conditions; d,e,f: comparison among all apo-CaM conditions; $p<0.05)$

not necessarily required for CaM binding [22]. Herein, we describe a novel incidence where phosphorylation regulates $\mathrm{CaM}$ binding to a target protein. In this case, the PKA-dependent phosphorylation of SMTNL1 at Ser301 modulates both $\mathrm{Ca}-$ and apo-CaM binding. Ser301 lies well upstream of the core hydrophobic residues within $\mathrm{CBD} 1$ required for $\mathrm{Ca}-\mathrm{CaM}$-binding (i.e., 20 amino acids from Phe321 which is the critical contributor [11]). It is likely that the distance between Ser301 and the hydrophobic patch of CBD1 is too distant for phosphorylation to directly block $\mathrm{Ca}-\mathrm{CaM}$ docking. This agrees with our observation that the rate of PKA-dependent phosphorylation at Ser301 was not influenced when apo- or $\mathrm{Ca}-\mathrm{CaM}$ was included in the kinase assay (unpublished results). We have previously demonstrated that intramolecular interactions between the $C$-terminal IQ motif (localization of the apo-CaM binding site, CBD2) and the intrinsically disordered region (localization of the phosphorylation site) influence apoCaM binding $[11,12]$. Furthermore, a portion of the $N$ terminal intrinsically disordered region forms intramolecular contacts with the globular $C$-terminal calponin homology $(\mathrm{CH})$ domain [18]. Thus, it is conceivable that phosphorylation within the $\mathrm{N}$-terminal region has farreaching effects in the $C$-terminal domain and can attenuate both $\mathrm{Ca}$ - and apo-CaM binding potentials.

The phosphorylation of SMTNL1-TMB enhanced the Tpm-binding potential in both pull down studies and by confirming SPR assays. Inconsequential amounts of nonspecific Tpm-binding were found with CaM-Sepharose, and its binding was not influenced by the presence of SMTNL1-TMB, irrespective of phosphorylation or calcium. Thus, we conclude that a heterotrimeric complex of SMTNL1-TMB, CaM and Tpm was not formed under any of the binding conditions employed. Since no heterotrimer was generated, we suggest that $\mathrm{Tpm}$ and $\mathrm{Ca}-\mathrm{CaM}$ compete for a similar binding site on SMTNL1-TMB. This conclusion is further supported by the fact that the binding surfaces for CaM and Tpm overlap on SMTNL1. Indeed, only very weak binding of Tpm is observed with the isolated $\mathrm{CH}$ domain (containing: CBD2; apo-CaM binding) in the absence of the intrinsically-disordered upstream sequence (containing: CBD1, Ca-CaM binding; and Ser301, phosphorylation consensus sequence) [18].

Our findings place SMTNL1 in a position to influence both $\mathrm{Ca}^{2+} / \mathrm{CaM}$-regulated contractile events and $\mathrm{Ca}^{2+}$ desensitization pathways in smooth muscle. Depending on intracellular calcium concentration and cyclic nucleotide-dependent kinase activation, SMTNL1 is predicted to cycle between Tpm-bound and Ca-CaMbound complexes. The impact of SMTNL1 on smooth muscle contractile processes may be via its binding partners and associations with the contractile filament. In its dephosphorylated state SMTNL1 has weaker affinity for $\mathrm{Tpm}$ and would be more likely to associate with CaM. With an increase in intracellular calcium due to a contractile signal, Ca-CaM could bind to SMTNL1 and initiate the dissociation of the complex from the thin filament. Unphosphorylated SMTNL1 can inhibit myosin light chain phosphatase (MLCP) activity in vitro [27], so the release of SMTNL1 could attenuate MLCPdependent dephosphorylation of myosin regulatory light chain (LC20). Conversely, SMTNL1 phosphorylation during calcium desensitization of smooth muscle could provide enhanced binding to Tpm and restrict binding of $\mathrm{CaM}$ in the apo-state, securing phosphorylated SMTNL1 with Tpm on the thin filament. The weak binding modes of SMTNL1 with apo-CaM might not possess enough stability to occur in vivo [12]. While the physiological significance of 

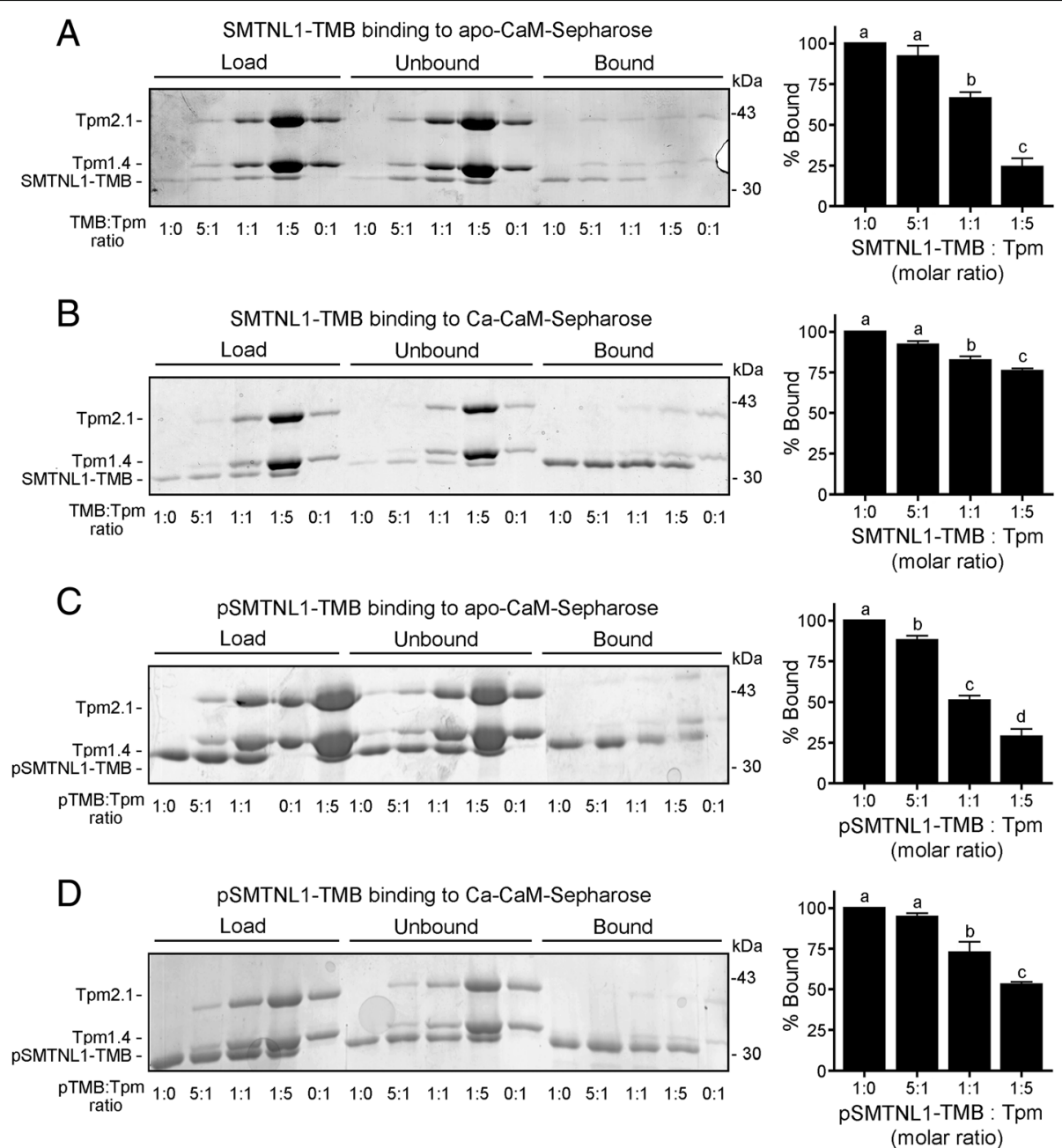

Fig. 3 SMTNL1 phosphorylation precludes Ca-CaM-binding in favour of Tpm-binding under conditions of high Tpm content. SMTNL1-TMB or phosphorylated SMTNL1-TMB (concentrations as described in Fig. 2) was pre-incubated with the indicated molar ratio of purified a/ $\beta$ Tpm dimer. The mixtures were then added to CaM-Sepharose in the absence (a, $\mathbf{c}$; apo-CaM, 1 mM EDTA), or presence (b, d; Ca-CaM, 5 mM CaCl 2$)$ of calcium. After extensive washing, bound SMTNL1-TMB was eluted and detected with Coomassie staining of SDS-PAGE gels. The band density was quantified and binding expressed as percentage of maximum binding within each condition. All experiments are $n=3$ and were analyzed by one-way ANOVA with Tukey's post hoc analysis. Different letters indicate significant differences among groups $(p<0.05)$

SMTNL1 association with the contractile filament in situ has not been investigated, we speculate that the protein may act in analogous manners to caldesmon or calponin on actin-myosin cross bridge cycling. Calponin interacts with many cytoskeleton and related proteins (including tropomyosin) and functions as an inhibitor of actinactivated myosin ATPase [28]. The binding of $\mathrm{Ca}-\mathrm{CaM}$ dissociates calponin from the actin filament to facilitate smooth muscle contraction [29]. Calponin can also be phosphorylated in response to cyclic nucleotide signals to cause a reduced affinity for the thin filament [30]. Caldesmon is another actin filament-associated regulatory protein; it also binds to Tpm and regulates cross-bridge cycling in a $\mathrm{Ca}-\mathrm{CaM}$ dependent manner [31]. A detailed physiological analysis of the CaM- and Tpm-interactions of SMTNL1 and their functional implications are required to provide a clear description of role of SMTNL1 in smooth muscle contractility. Further investigation will be needed to verify the role of SMTNL1 in fine-tuning thin filament dynamics.

\section{Conclusions}

Herein, we provide evidence that calcium availability increases CaM association with SMTNL1, and CaM and tropomyosin binding to SMTNL1 are mutually exclusive events. Moreover, SMTNL1 binding to CaM is reduced by phosphorylation of Ser301 while binding to tropomyosin is enhanced. We propose a model whereby SMTNL1 can exist in distinct complexes with either Tpm or CaM, depending on availability of calcium and activity of PKA. This 
switching mechanism could aid in the fine-tuning of smooth muscle contraction.

\section{Additional file}

Additional file 1: Figure S1. SPR measurements for binding of SMTNL1-TMB to tropomyosin. Sensorgrams and data fittings are provided for unphosphorylated and phosphorylated (S301 with PKA) SMTNL1-TMB protein with tropomyosin immobilized via amine-coupling to a CM5 sensor chip. (DOCX 714 kb)

\section{Abbreviations}

apo-CaM: Calcium-free calmodulin; Ca-CaM: Calcium-saturated calmodulin; CaM: Calmodulin; CBD1 and CBD2: Calmodulin binding domain 1 and 2, respectively; PKA: Protein kinase A; PKG: Protein kinase G; SMTNL1: Smoothelin-like 1; SMTNL1-TMB: Tropomyosin-binding region of SMTNL1; Tpm: Tropomyosin

\section{Acknowledgements}

Not applicable.

\section{Funding}

This work was supported by the Canadian Institutes of Health Research (MOP-97931). JAM held an Alberta Innovates - Health Solutions (AlHS) Senior Scholar Award and was recipient of a Canada Research Chair (Tier 2) in Smooth Muscle Pathophysiology. AUL was a Heart \& Stroke Foundation of Canada Postdoctoral Fellow.

\section{Availability of data and materials}

All data generated and/or analysed during this study are included in the published article or its Additional file 1.

\section{Authors' contributions}

AUL, HI and DHS designed, performed and analyzed the majority of experiments. AUL and JAM wrote the manuscript and drafted figures. JAM and HJV conceived and coordinated the study. All authors reviewed the results and approved the final version of the manuscript.

\section{Competing interests}

The authors declare that they have no competing interests.

\section{Consent for publication}

Not applicable.

\section{Ethics approval and consent to participate}

All experiments utilizing animal tissues were approved by the Health Sciences Animal Care Committee, University of Calgary and conform to the guidelines set by the Canadian Council on Animal Care.

\section{Publisher's Note}

Springer Nature remains neutral with regard to jurisdictional claims in published maps and institutional affiliations.

\section{Author details}

'Department of Biochemistry \& Molecular Biology, University of Calgary, Cumming School of Medicine, 3280 Hospital Drive NW, Calgary, AB T2N 4Z6, Canada. ${ }^{2}$ Biochemistry Research Group, Department of Biological Sciences, University of Calgary, 2500 University Drive NW, Calgary, AB T2N 1 N4, Canada

Received: 19 October 2016 Accepted: 15 March 2017

Published online: 21 March 2017

\section{References}

1. Borman MA, MacDonald JA, Haystead TA. Modulation of smooth muscle contractility by CHASM, a novel member of the smoothelin family of proteins. FEBS Lett. 2004;573:207-13.
2. Lontay B, Bodoor K, Sipos A, Weitzel DH, Loiselle D, et al. Pregnancy and smoothelin-like protein 1 (SMTNL1) deletion promote the switching of skeletal muscle to a glycolytic phenotype in human and mice. J Biol Chem. 2015:290:17985-98.

3. Bodoor K, Lontay B, Safi R, Weitzel DH, Loiselle D, et al. Smoothelin-like 1 protein is a bifunctional regulator of the progesterone receptor during pregnancy. J Biol Chem. 2011;286:31839-51.

4. Lontay B, Bodoor K, Weitzel DH, Loiselle D, Fortner C, et al. Smoothelin-like 1 protein regulates myosin phosphatase-targeting subunit 1 expression during sexual development and pregnancy. J Biol Chem. 2010;285:29357-66.

5. Wooldridge AA, Fortner CN, Lontay B, Akimoto T, Neppl RL, et al. Deletion of the protein kinase A/protein kinase G target SMTNL1 promotes an exerciseadapted phenotype in vascular smooth muscle. J Biol Chem. 2008:283:11850-9.

6. Turner SR, MacDonald JA. Novel contributions of the smoothelin-like 1 protein in vascular smooth muscle contraction and its potentia involvement in myogenic tone. Microcirculation. 2014;21:249-58.

7. Chin D, Means AR. Calmodulin: a prototypical calcium sensor. Trends Cell Biol. 2000;10:322-8.

8. Villarroel A, Taglialatela M, Bernardo-Seisdedos G, Alaimo A, Agirre J, et al. The ever changing moods of calmodulin: how structural plasticity entails transductional adaptability. J Mol Biol. 2014;426:2717-35.

9. Ishida H, Vogel HJ. Protein-peptide interaction studies demonstrate the versatility of calmodulin target protein binding. Protein Pept Lett. 2006;13: 455-65.

10. Ishida H, Borman MA, Ostrander J, Vogel HJ, MacDonald JA. Solution structure of the calponin homology $(\mathrm{CH})$ domain from the smoothelin-like 1 protein: a unique apocalmodulin-binding mode and the possible role of the C-terminal type-2 $\mathrm{CH}$-domain in smooth muscle relaxation. J Biol Chem. 2008;283:20569-78.

11. Ulke-Lemée A, Ishida H, Chappellaz M, Vogel HJ, MacDonald JA. Two domains of the smoothelin-like 1 protein bind apo- and calcium-calmodulin independently. Biochim Biophys Acta. 1844;2014:1580-90.

12. Ulke-Lemée A, Turner SR, MacDonald JA. In situ analysis of smoothelin-like 1 and calmodulin interactions in smooth muscle cells by proximity ligation. J Cell Biochem. 2015;116:2667-75.

13. Gunning PW, O'Neill G, Hardeman E. Tropomyosin-based regulation of the actin cytoskeleton in time and space. Physiol Rev. 2008;88:1-35.

14. Wang $\mathrm{CL}$, Coluccio LM. New insights into the regulation of the actin cytoskeleton by tropomyosin. Int Rev Cell Mol Biol. 2010;281:91-128.

15. Gunning PW, Hardeman EC, Lappalainen P, Mulvihill DP. Tropomyosin master regulator of actin filament function in the cytoskeleton. J Cell Sci. 2015:128:2965-74

16. Geeves MA, Hitchcock-DeGregori SE, Gunning PW. A systematic nomenclature for mammalian tropomyosin isoforms. J Muscle Res Cell Motil. 2015:36:147-53.

17. Rao JN, Rivera-Santiago R, Li XE, Lehman W, Dominguez R. Structural analysis of smooth muscle tropomyosin alpha and beta isoforms. J Biol Chem. 2012;287:3165-74

18. MacDonald JA, Ishida H, Butler El, Ulke-Lemée A, Chappellaz M, et al. Intrinsically disordered $\mathrm{N}$-terminus of calponin homology-associated smooth muscle protein (CHASM) interacts with the calponin homology domain to enable tropomyosin binding. Biochemistry. 2012;51:2694-705.

19. Ulke-Lemée A, Ishida H, Borman MA, Valderrama A, Vogel HJ, MacDonald JA. Tropomyosin-binding properties of the CHASM protein are dependent upon its calponin homology domain. FEBS Lett. 2010;584:3311-6.

20. Rannels SR, Beasley A, Corbin JD. Regulatory subunits of bovine heart and rabbit skeletal muscle cAMP-dependent protein kinase isozymes. Methods Enzymol. 1983;99:55-62.

21. Jancso A, Graceffa P. Smooth muscle tropomyosin coiled-coil dimers. Subunit composition, assembly, and end-to-end interaction. J Biol Chem. 1991;266:5891-7.

22. Lukas TJ, Burgess WH, Prendergast FG, Lau W, Watterson DM. Calmodulin binding domains: characterization of a phosphorylation and calmodulin binding site from myosin light chain kinase. Biochemistry. 1986;25:1458-64.

23. Graff JM, Young TN, Johnson JD, Blackshear PJ. Phosphorylation-regulated calmodulin binding to a prominent cellular substrate for protein kinase $\mathrm{C}$. J Biol Chem. 1989;264:21818-23.

24. Hofmann F, Anagli J, Carafoli E, Vorherr T. Phosphorylation of the calmodulin binding domain of the plasma membrane Ca2+ pump by protein kinase $C$ reduces its interaction with calmodulin and with its pump receptor site. J Biol Chem. 1994;269:24298-303. 
25. Fleming I, Fisslthaler B, Dimmeler S, Kemp BE, Busse R. Phosphorylation of Thr(495) regulates $\mathrm{Ca}(2+) /$ calmodulin-dependent endothelial nitric oxide synthase activity. Circ Res. 2001;88:E68-75.

26. Wong $R$, Schlichter LC. PKA reduces the rat and human KCa3.1 current, CaM binding, and $\mathrm{Ca} 2+$ signaling, which requires Ser332/334 in the CaM-binding C terminus. J Neurosci. 2014;34:13371-83.

27. Borman MA, Freed TA, Haystead TA, MacDonald JA. The role of the calponin homology domain of smoothelin-like 1 (SMTNL1) in myosin phosphatase inhibition and smooth muscle contraction. Mol Cell Biochem. 2009;327:93-100.

28. Liu R, Jin JP. Calponin isoforms CNN1, CNN2 and CNN3: Regulators for actin cytoskeleton functions in smooth muscle and non-muscle cells. Gene. 2016;585: $143-53$.

29. Naka M, Kureishi Y, Muroga Y, Takahashi K, Ito M, Tanaka T. Modulation of smooth muscle calponin by protein kinase $\mathrm{C}$ and calmodulin. Biochem Biophys Res Commun. 1990;171:933-7.

30. Winder SJ, Walsh MP. Smooth muscle calponin. Inhibition of actomyosin MgATPase and regulation by phosphorylation. J Biol Chem. 1990;265: 10148-55.

31. Marston SB, Redwood CS. The essential role of tropomyosin in cooperative regulation of smooth muscle thin filament activity by caldesmon. J Biol Chem. 1993;268:12317-20.

\section{Submit your next manuscript to BioMed Central} and we will help you at every step:

- We accept pre-submission inquiries

- Our selector tool helps you to find the most relevant journal

- We provide round the clock customer support

- Convenient online submission

- Thorough peer review

- Inclusion in PubMed and all major indexing services

- Maximum visibility for your research

Submit your manuscript at www.biomedcentral.com/submit 